\title{
Advance Care Planning in Healthcare Systems: A Literature Review and Call to Action
}

\author{
Laura Finn $\mathrm{MD}^{1^{*}}$, Juliette Folse $\mathrm{RN}^{1}$, Melissa Piglia RN ${ }^{1}$, Kathy Pirozzolo RN ${ }^{1}$, Michelle \\ Thomas $\mathbf{R N}^{2}$, Lorrie Erario $\mathbf{R N}^{3}$ \\ ${ }^{1}$ Division of Hematology and Bone Marrow Transplant. Department of Internal Medicine. Ochsner Health \\ Foundation. 1514 Jefferson Highway, New Orleans, Louisiana 70121, United States \\ ${ }^{2}$ Division of Oncology. Department of Internal Medicine. Ochsner Health Foundation. 1514 Jefferson \\ Highway, New Orleans, Louisiana 70121, United States
}

${ }^{3}$ Division of Oncology, Hematology and Bone Marrow Transplant. Department of Internal Medicine. Ochsner Health Foundation. 1514 Jefferson Highway, New Orleans, Louisiana 70121, United States

*Corresponding Author: Laura Finn, Division of Hematology and Bone Marrow Transplant. Department of Internal Medicine. Ochsner Health Foundation. 1514 Jefferson Highway, New Orleans, Louisiana 70121, United States. Email: laura.finn@ochsner.org

\begin{abstract}
Advance care planning is a vital part of healthcare delivery concordant with patient wishes. The practice of advance care planning entails patient-provider conversations regarding treatment wishes including end-of-life care, documentation of these decisions by advance directives, and action upon such decisions at the appropriate time. Despite patient and provider appreciation of advance care planning, the practice remains under-utilized. In this review we discuss the history and benefits of advance care planning. We describe the features of efficient practice of advance care planning and perceived barriers that need surpassing. Finally, we discuss potential clinical pathways, the need for research in advance care planning, and stakeholder action needed to promote advance care planning across healthcare systems.
\end{abstract}

Keywords: Advance care planning, advance directive, clinical pathways, electronic medical record, palliative medicine

\section{INTRODUCTION}

The international consensus definition of advance care planning (ACP) is "enabling individuals who have decision making capacity to identify their values, to reflect upon the meanings and consequences of serious illness scenarios, to define goals for future medical treatment and care, and to discuss these with family and health care providers. ACP addresses individual's concerns across the physical, psychological, social, and spiritual domains. It encourages individuals to identify a personal representative and to record and regularly review any preferences, so that their preferences can be considered should they at some point be unable to make their own decisions." [1] The history of ACP in the United States is often viewed by the politics of advance directives. Advance directives are the legal forms completed to declare specific ACP decisions including the healthcare power (HCPOA) of attorney and living will. End-of-life care orders, according to patient wishes, that are ordered by a healthcare provider include a do not resuscitate order (DNR) and physician order for life sustain therapy (POLST).

The legal history of advance directives begins with the case of Karen Quinlan in 1976. Karen had a cardiopulmonary arrest at 21-years-old. She was resuscitated, intubated, feeding tube was placed, and never regained consciousness. A year later physicians refused to discontinue ventilation for fear of legal retribution; therefore, the New Jersey Supreme Court hearing her parents testify she would not want to exist in this state ruled that a competent person has the right to refuse life-sustaining therapy and this right is not lost when they cannot speak for themselves. [2] This case led to state by state development of the living will and provided legal protection to physicians who honor patients' written advance directives.

The case of Nancy Cruzan made advance directives constitutional law by the US Supreme 
Court in 1990. Nancy at 25-years-old was involved in a motor vehicle accident. She was resuscitated but remained in a persistent vegetative state. Her life was maintained by feeding tube and artificial nutrition. The Missouri Supreme Court ruled that her feeding tube could only be discontinued if she made that decision or her family could provide "clear and convincing" evidence it was her wish. The subsequent US Supreme Court decision was absent of controversy. By the US constitution, Americans have always had the right to refuse any medical intervention, including intubation, ventilation, and artificial delivery of nutrition and hydration.[3] This decision led to passage of the Patient Self Determination Act mandating all medical institutions inform patients of their right to have advance directives.[4]

ACP at its core is a set of essential conversations that every adult needs to have with their physicians and their trusted family and/or friends. These cases of healthy 20 -year-old adults who fell into sudden vegetative states highlight the fact that all adults, at any age or state of health, need to consider, verbalize, and document their true beliefs for care during serious illness including the end-of-life and who they want to express those wishes should they be unconscious or unable. When chronic or progressive health conditions do occur, advance care planning discussions may become more direct and should be updated as health and prognosis evolve. In this review we discuss the current state of practice and use of ACP and advance directives. We evaluate the current barriers to this practice in health care systems. We also describe the features of successful programs and consider methods for improvement and areas for future research.

\section{Materials ANd Methods}

A literature review was performed for key words including ACP, advance directives, health care power of attorney, living, will, do not resuscitate, physician orders for life sustaining therapy, palliative care, palliative medicine, end-of-life, shared decision making, and clinical care pathways. The literature was also reviewed for themes in ACP including current use and perceived benefits, features of successful ACP programs, barriers to ACP, clinical care pathways, and the state of research in ACP. The literature was also reviewed for current guidelines, payor statements, and professional society recommendations for ACP.

\section{ReSUltS}

\subsection{Benefits of Advance Care Planning}

ACP is an opportunity to promote shared decision making regarding unexpected or difficult medical situations amongst patients, their family members, caregivers and their healthcare providers. Current evidence from systematic reviews show that ACP results in care concordant with patient wishes, improves consistency of care, and increases completion of advance directives. Patient satisfaction and quality of life increases with ACP. A review of patient perceptions regarding ACP found patients have more confidence about end-of-life care and felt more peace and control after ACP discussions.[5] A review of studies published between 2011-2016 estimates that $36.7 \%$ of US adults have an advance directive. This finding was similar amongst healthy adults and those with chronic illness.[6]

ACP improves the quality of patient-provider communication.[7, 8] Decision control preference (DCP) is the amount of control patents want to have over their medical decisions. In culturally diverse populations these preferences can vary greatly from desire for full patient control, shared decision making, or clinicians make all decisions. A cross-sectional survey of adults with co-morbid conditions found despite preconceptions, adults with low DCP readily engaged in ACP, goals of care decisions and maintained confidence in their communication with physicians.[9] One of the reasons for improved patient-provider communication, is the fact providers acquire improved communication skills by ACP planning. ACP conversations may be spontaneous, but trained providers often go through a 4-step planning phase as outlined by a 2018 review of these conversations. Providers first identify patients and their circumstances applicable for discussion, plan the conversation, explore patient perspectives, and act on the decisions made, including advance directives, planning acceptable treatments, and reducing hospital admissions.[10]

Studies of a variety of patient populations show that ACP does in fact reduce unnecessary hospitalizations. [7, 11-13] This includes patients outside of the hospital or clinic setting. Nursing home patients with ACP reduce avoidable hospitalizations.[14] Patients with ACP have reduced cost of care driven by these reduced hospitalizations. A study of Medicare beneficiaries with a history of ACP found their cost of care was $\$ 9,500$ less than those without 
ACP due to decreased hospitalizations and shorter inpatient stays. [15] Through appropriate use of palliative care and ACP, health care systems can save nearly 2 million dollars annually through reduced length of inpatient stays and daily hospital costs. [16, 17]

ACP increases the use of Palliative Medicine, giving patients access to specialists of ACP, treating refractory symptoms of chronic illnesses, setting goals of care, and managing all end of life issues. [18] A multicenter randomized control trial of interdisciplinary Palliative Medicine versus usual hospital care for patients with terminal illnesses resulted in enhancements of the benefits already seen with ACP including improved care experiences, better provider communication, less ICU admissions, and lower total cost of healthcare. Appropriately, the patients randomized to Palliative Medicine also had the benefit of hospice admission durations twice as long as the control group.[19] Early referrals to Palliative Medicine increase care delivery concordant with patient wishes, improving the quality of life for both patients and their families.[20, 21]

\subsection{Successful ACP Implementation in Healthcare Systems}

Successful implementation of ACP in health care organizations requires an imbedded infrastructure including consistent workflow, standardized documents, and utilization of electronic medical records (EMR) including patient portals, organizational support and guidance, and inclusion of ACP metrics as part of clinical governance requirements. [22] Results of randomized control trials are clear, patients in intervention groups participate in ACP and complete advance directives more often than control groups [23] emphasizing the fact that system-wide interventional approaches to ACP are effective. Two of the most vital components of health system ACP implementation are systematic facilitation of ACP conversation and effectively documenting these conversations in the EMR for easy access across the system. [24]

A significant amount of health system effort is exerted in training multiple levels of healthcare providers to identify patients and facilitate ACP conversations. [25, 26] This workflow may begin with patient intake and inquiry about current advance directives at every visit. Nursing staff initiate conversations or even facilitate formal ACP discussions. [27, 28] A large community oncology practice evaluated a system approach to ACP using nursing staff education and interventions and developed EMR documentation resulting in a 2-fold increase in ACP with patients. [29] Facilitators of ACP first assess patient knowledge of ACP, build up motivation to discuss current care and end of life care wishes, then facilitate mutual decision regarding care preferences with or without completing advance directives. [25] System approaches to ACP must cater to individual needs and a variety of patients. Younger patients may need broad ACP education while elderly patients, especially those who are frail will have higher acuity and urgency for ACP. [30]

The EMR is a tool to facilitate ACP by providing easy identification of patients without advance directives and documentation of ACP conversations and decisions. A review of the utility of the EMR in ACP showed its most common uses are establishing documentation templates, creating electronic order sets, and automated prompts to trigger ACP discussions. [31] The value of using the EMR in this setting is not only to prompt ACP activity but also allow all providers to find the ACP information stored in the record. This area of EMR utility needs improvement. An analysis of Emergency Room physicians that need rapid access to care decisions found only a third felt confident they could find ACP documentation in their EMR. [32] One solution to increase ease of access to ACP documentation is creation of a "home" in the EMR. Called a "Care Directives Activity Tab" by one study, this designates a singlelocation in the EMR for storing and viewing all ACP activities, shown to increase ACP use and documentation when available. [33, 34] In addition to facilitating ACP activities, the EMR may also be used to follow a healthcare system's usage of ACP through tracking advance directive documents or billing codes of ACP activity. [35] Tracking ACP activity allows healthcare systems to identify areas at need for additional ACP advocacy.

\subsection{Gaps in Advocacy}

The most upfront barrier to ACP is provider mindset that it is only end-of-life care planning. The history of ACP and advance directives outlines the need for ACP at the onset of decision-making capacity for any young adult. The 2014 updated Institute of Medicine Dying in America report outlines a life-cycle of ACP that starts with adulthood, decisions are revisited as acute and chronic health conditions evolve during our life span and completes with end-oflife decisions and dying. [36] International 
recognition of this age bias to initiating $\mathrm{ACP}$ has led to increasing movements to normalize death conversations with young, healthy patients to promote health living and develop realistic relationships with disease and dying. [37, 38]

Another barrier to ACP is the tendency for providers to associate the need for ACP with patient prognosis. This behavior is wrought with error. This practice excludes most of our adult population who are healthy and absent of chronic illness. In addition providers, including specialists, are unable to estimate prognosis due to inaccurate optimism. [39] Provider decisions to discuss ACP vary across chronic illnesses with similar prognosis. In a retrospective cohort study of three teaching hospitals and a Veterans Administration hospital, terminally ill patients with acquired immunodeficiency (AIDS), unresectable non-small cell lung cancer, severe cirrhosis, and severe congestive heart failure were reviewed for advance directives. Do not resuscitate (DNR) orders were placed for $52 \%$ of patients with AIDS, $47 \%$ with lung cancer, $16 \%$ with cirrhosis, and $5 \%$ with heart failure despite all patients having a similar poor prognosis. [40] Additional review of DNR orders in hospitalized patients continue to emphasize patient comorbidities rather than underlying prognosis, with cancer being the primary diagnosis to initiate the DNR conversation. [41, 42] Interestingly, surveys of clinician perspectives of barriers of ACP in hospitalized patients and oncology clinics found that clinicians do not recognize their own limitations. Instead the perceived patients and their families did not accept their own poor prognosis. [43, 44] Providers also describe inherent difficult in discussing ACP and advance directives with patients pursuing curative therapies, research describes this phenomenon in patients awaiting both solid organ and bone marrow transplants. $[45,46]$

The description of ACP barriers described by clinicians has recurring themes of poor understanding of prognosis, ignorance of the limitations of life-sustaining therapies, and lack of capacity to understand goals of care conversations on the part of patients, families, and caregivers. [43, 44, 47] Ironically, this is not likely the result of poor understanding by patients and their families regarding the role of ACP and use of advance directives. This is the result of initiating ACP too late in course of a chronic and eventual terminal illness. The studies of these clinician perceptions are reviews of physicians treating cancer, severe heart disease, and hospitalized patients. This perceived barrier to ACP on part of patients can be overcome by earlier ACP discussions and early referral to Palliative Medicine. [48, 49]

\subsection{Methods for Improvement/ Pathway Development}

Healthcare providers require conversation training for successful ACP. ACP education increases provider knowledge, comfort, and attitude toward participating in challenging conversations. With training and process improvements physicians and nurses can routinely implement ACP in routine, nonpalliative care settings. It is important to invest in training for the full spectrum of healthcare providers while realizing that providers in acute care and inpatient settings may have unique challenges to ACP. Nurses in acute care settings tend to have lower involvement in ACP siting lack of time and acute symptom management as barriers, in addition to lack of education and training. [50, 51] Adequate staff training is the primary step in improving and implementing ACP as the presence of prepared staff is critical to operationalize ACP in a health care system. [22] Regular in-service, skills workshops, and role-play should be a part of training new clinical staff and refreshing the skills of an organizations ACP workforce. [52] The healthcare staff should also be encouraged to consider and complete their own advance directives.

Structured communication tools are available for conversation training and have been shown to increase ACP. [53] Available training curriculums for ACP facilitators and physician coaching are available. Two examples are Vital Talk ${ }^{\circledR}$ and Respecting Choices ${ }^{\circledR}$. A study of implementation of this training with physicians and nurses in a primary care practice found the organization increase from zero ACP conversations at baseline to 7589 conversations over a year with $29 \%$ of conversations resulting in placement of advance directives in the EMR. [54] The Accreditation Council for Graduate Medical Education, the oversight organization of resident and fellow education, now requires palliative medicine and ACP in resident training. Residents and fellows are now often leaders in ACP in academic healthcare settings. Trainees report confidence with ACP after education 
sessions and novel approaches to resident training, such as using medical and legal educators for teaching. This is an area full of research potential. [55]

As previously reported, Palliative Medicine and ACP are lacking in clinical care pathway development. [56] A definition of a care pathway is "a methodology for mutual decision making of care for a well-defined group of patients during a well-defined period." [57] A clinical care pathway includes a specific statement of goals based on evidence and patient expectations. The pathway also includes descriptions of stakeholder roles and the sequence of activities that will take place. Clinical care pathways standardize the approach to a clinical problem allowing for measurement of outcome metrics, facilitating research and quality improvement of ACP programming. [56] Development of a clinical care pathway is a mechanism for healthcare systems to standardize ACP across their organizations and to unify clinical practice with guidelines and professional society recommendations for ACP.

\section{DISCUSSION AND RECOMMENDATIONS}

All adults and even children with appropriate capacity should participate in their healthcare decisions throughout their lives and as end of life approaches to receive care consistent with their values and goals. All healthcare providers have the responsibility to initiate these conversations to integrate patient preferences into care planning. [36] ACP has well documented benefits as discussed in this review; however, to date there are no practice guidelines broadly applicable to a variety of cultural setting and personal values. This hinders the broad development of ACP programs and evaluation of ACP use and effectiveness in healthcare systems that cater to large patient populations. [1] A survey of community, federal, and academic healthcare systems found that the spectrum of interprofessional team members play a role in ACP including nurses, advanced practice providers, social workers, chaplains, and physicians. $62 \%$ of respondents to the survey were unaware of ACP policies at their institutions. Two-thirds of those surveyed agreed that ACP is a high priority and training for providers and dedicated staff to facilitate ACP is needed. [58]
Payors and healthcare system administrations need to integrate quality surveillance measures of organizational level ACP. Payors and health systems should harmonize organizational ACP programs with financial incentives, including costs saved by decreasing hospitalization, emergency room visits, intensive care and acute care visits. Health systems should also invest in EMR programming that incorporates ACP across all types of patient encounters and providers. [36] Professional societies and other organizations that establish quality standards should develop clear policy statements for clinician-patient communication and ACP that are measurable, actionable, and evidence-based. Fortunately, this call to action is becoming an expectation of some payors and professional societies. The Centers for Medicare and Medicaid Oncology Care Model for practice and reimbursement requires ACP within the first three visits for new patients in the outpatient setting. [59] The American Society of Oncology Quality Oncology Practice Initiative requires documentation of patient's advance directives by their third outpatient visit as a measure of quality care. [60]

Further research is needed to improve the practice and implementation of ACP. Most ACP research which has occurred since 2014, is low quality and tends to cover specific topics of palliative care interventions, decision-making, communication strategies, factors of implementation, effectiveness, and cost.[61] More research is needed on the holistic approach of interdisciplinary teams, impact of all stakeholders including payors and healthcare system administration, development of clinical care pathways, and the public health perspective of ACP. Higher quality studies are needed including randomized control trials and innovative strategies to approach a range of populations and cultures with ACP to address these current research gaps.

\section{CONCLUSiON}

ACP is acknowledged and respected by healthcare providers and patients; however, barriers and bias to ACP are active impediments in daily clinical practice. Healthcare systems and organizations can overcome these barriers by staff training, development of clinical care pathways, and administrative support of ACP activities. Large, randomized, and preferably multi-institutional clinical trials are needed to build a stronger body of evidence for ACP, provide population level results, and to continue advocacy for all patients for ACP. 


\section{REFERENCES}

[1] Rietjens, J.A.C., et al., Definition and recommendations for advance care planning: an international consensus supported by the European Association for Palliative Care. Lancet Oncol, 2017. 18(9): p. e543-e551.

[2] Coburn, D.R., In re Quinlan: A Practical Overview. Ark. L. Rev., 1977. 31: p. 59.

[3] v Director, C., Missouri Dept of Health. 497 US, 1990: p. 261.

[4] Greco, P.J., et al., The Patient SelfDetermination Act and the future of advance directives. Annals of Internal Medicine, 1991. 115(8): p. 639-643.

[5] Hall, A., C. Rowland, and G. Grande, How Should End-of-Life Advance Care Planning Discussions Be Implemented According to Patients and Informal Carers? A Qualitative Review of Reviews. J Pain Symptom Manage, 2019. 58(2): p. 311-335.

[6] Yadav, K.N., et al., Approximately One In Three US Adults Completes Any Type Of Advance Directive For End-Of-Life Care. Health Aff (Millwood), 2017. 36(7): p. 1244-1251.

[7] Au, D.H., et al., A Randomized Trial to Improve Communication About End-of-Life Care Among Patients With COPD. Chest, 2012. 141(3): p. 726-735.

[8] Murray, M.A., et al., Skills Training to Support Patients considering place of End-Of-Life Care: A Randomized Control Trial. Journal of Palliative Care, 2010. 26(2): p. 112-121.

[9] Chiu, C., et al., "Doctor, Make My Decisions": Decision Control Preferences, Advance Care Planning, and Satisfaction With Communication Among Diverse Older Adults. Journal of pain and symptom management, 2016. 51(1): p. 3340 .

[10] Fahner, J.C., et al., Interventions Guiding Advance Care Planning Conversations: A Systematic Review. J Am Med Dir Assoc, 2019. 20(3): p. 227-248.

[11] O'Halloran, P., et al., Advance Care Planning With Patients Who Have End-Stage Kidney Disease: A Systematic Realist Review. Journal of pain and symptom management, 2018. 56(5): p. 795-807.e18.

[12] Song, K., et al., Advance Care Planning in Patients with Primary Malignant Brain Tumors: A Systematic Review. Front Oncol, 2016. 6: p. 223.

[13] Kernick, L.A., et al., Does advance care planning in addition to usual care reduce hospitalisation for patients with advanced heart failure: A systematic review and narrative synthesis. Palliat Med, 2018. 32(10): p. 15391551.
[14] Hickman, S.E., et al., Systematic Advance Care Planning and Potentially Avoidable Hospitalizations of Nursing Facility Residents. Journal of the American Geriatrics Society, 2019. 67(8): p. 1649-1655.

[15] F., B.W., et al., Advance Care Planning in an Accountable Care Organization Is Associated with Increased Advanced Directive Documentation and Decreased Costs. Journal of Palliative Medicine, 2018. 21(4): p. 489-502.

[16] Cowan, J.D., Hospital charges for a community inpatient palliative care program. Am J Hosp Palliat Care, 2004. 21(3): p. 177-90.

[17] Ciemins, E.L., et al., The economic and clinical impact of an inpatient palliative care consultation service: a multifaceted approach. $\mathrm{J}$ Palliat Med, 2007. 10(6): p. 1347-55.

[18] Hanson, A.P.L.C., et al., A Quality Improvement Intervention to Increase Palliative Care in Nursing Homes. Journal of Palliative Medicine, 2005. 8(3): p. 576-584.

[19] Gade, G., et al., Impact of an Inpatient Palliative Care Team: A Randomized Controlled Trial. Journal of Palliative Medicine, 2008. 11(2): p. 180-190.

[20] Silveira, M.J., S.Y.H. Kim, and K.M. Langa, Advance Directives and Outcomes of Surrogate Decision Making before Death. New England Journal of Medicine, 2010. 362(13): p. 12111218.

[21] Finn, L., A.R. Green, and S. Malhotra, Oncology and Palliative Medicine: Providing Comprehensive Care for Patients With Cancer. The Ochsner journal, 2017. 17(4): p. 393-397.

[22] Lund, S., A. Richardson, and C. May, Barriers to advance care planning at the end of life: an explanatory systematic review of implementation studies. Plos one, 2015. 10(2): p. e0116629.

[23] Houben, C.H.M., et al., Efficacy of advance care planning: a systematic review and metaanalysis. J Am Med Dir Assoc, 2014. 15(7): p. 477-489.

[24] Reidy, J., et al., Health System Advance Care Planning Culture Change for High-Risk Patients: The Promise and Challenges of Engaging Providers, Patients, and Families in Systematic Advance Care Planning. J Palliat Med, 2017. 20(4): p. 388-394.

[25] Lin, C.-P., et al., The conceptual models and mechanisms of action that underpin advance care planning for cancer patients: A systematic review of randomised controlled trials. Palliative medicine, 2019. 33(1): p. 5-23.

[26] MacKenzie, M.A., et al., Respecting Choices and Related Models of Advance Care Planning: A Systematic Review of Published Evidence. Am J Hosp Palliat Care, 2018. 35(6): p. 897-907. 
[27] Moore, C.D., Communication issues and advance care planning. Semin Oncol Nurs, 2005. 21(1): p. 11-9.

[28] Bushinski, R.L. and K.M. Cummings, Practices of effective end-of-life communication between nurses and patients/families in two care settings. Creat Nurs, 2007. 13(3): p. 9-12.

[29] Mikan, S.Q., et al., Improvements in communication and engagement of advance care planning in adults with metastatic cancer through a targeted team approach. Journal of Clinical Oncology, 2016. 34(26_suppl): p. 1414.

[30] Combes, S., et al., Implementing advance care planning with community-dwelling frail elders requires a system-wide approach: An integrative review applying a behaviour change model. Palliative medicine, 2019. 33(7): p. 743756.

[31] Huber, M.T., et al., Utilizing the Electronic Health Record to Improve Advance Care Planning: A Systematic Review. Am J Hosp Palliat Care, 2018. 35(3): p. 532-541.

[32] Lakin, J.R., et al., Emergency Physicians' Experience with Advance Care Planning Documentation in the Electronic Medical Record: Useful, Needed, and Elusive. J Palliat Med, 2016. 19(6): p. 632-8.

[33] Turley, M., et al., Impact of a Care Directives Activity Tab in the Electronic Health Record on Documentation of Advance Care Planning. Perm J, 2016. 20(2): p. 43-8.

[34] Walker, E., et al., Advance Care Planning Documentation Practices and Accessibility in the Electronic Health Record: Implications for Patient Safety. J Pain Symptom Manage, 2018. 55(2): p. 256-264.

[35] Dingfield, L.E. and J.B. Kayser, Integrating Advance Care Planning Into Practice. Chest, 2017. 151(6): p. 1387-1393.

[36] Ness, D.L. and B.H. Johnson, Dying in America: a constructive step forward and an opportunity to deepen partnerships with patients and families. Ann Intern Med, 2015. 162(3): p. 2267.

[37] Llewellyn, R., et al., Living into death: a case for an iterative, fortified and cross-sector approach to advance care planning. Anthropol Med, 2017. 24(3): p. 350-365.

[38] Bernacki, R.E. and S.D. Block, Communication about serious illness care goals: a review and synthesis of best practices. JAMA Intern Med, 2014. 174(12): p. 1994-2003.

[39] Christakis, N.A. and E.B. Lamont, Extent and determinants of error in physicians' prognoses in terminally ill patients: prospective cohort study. The Western journal of medicine, 2000. 172(5): p. 310-313.
[40] Wachter, R.M., et al., Decisions about resuscitation: inequities among patients with different diseases but similar prognoses. Ann Intern Med, 1989. 111(6): p. 525-32.

[41] Osinski, A., et al., Do-not-resuscitate orders in cancer patients: a review of literature. Support Care Cancer, 2017. 25(2): p. 677-685.

[42] de Decker, L., et al., Do not resuscitate orders and aging: impact of multimorbidity on the decision-making process. J Nutr Health Aging, 2014. 18(3): p. 330-5.

[43] You, J.J., et al., Barriers to goals of care discussions with seriously ill hospitalized patients and their families: a multicenter survey of clinicians. JAMA Intern Med, 2015. 175(4): p. 549-56.

[44] Piggott, K.L., et al., Breaking silence: a survey of barriers to goals of care discussions from the perspective of oncology practitioners. BMC Cancer, 2019. 19(1): p. 130.

[45] Potosek, J., et al., Integration of palliative care in end-stage liver disease and liver transplantation. Journal of palliative medicine, 2014. 17(11): p. 1271-1277.

[46] Loggers, E.T., et al., Advance care planning among hematopoietic cell transplant patients and bereaved caregivers. Bone Marrow Transplant, 2014. 49(10): p. 1317-22.

[47] You, J.J., et al., Barriers to Goals of Care Discussions With Patients Who Have Advanced Heart Failure: Results of a Multicenter Survey of Hospital-Based Cardiology Clinicians. J Card Fail, 2017. 23(11): p. 786-793.

[48] Temel, J.S., et al., Early palliative care for patients with metastatic non-small-cell lung cancer. N Engl J Med, 2010. 363(8): p. 733-42.

[49] Kavalieratos, D., et al., Association between palliative care and patient and caregiver outcomes: A systematic review and metaanalysis. JAMA, 2016. 316(20): p. 2104-2114.

[50] Rietze, L. and K. Stajduhar, Registered nurses' involvement in advance care planning: an integrative review. International journal of palliative nursing, 2015. 21(10): p. 495-503.

[51] Duke, G. and S. Thompson, Knowledge, attitudes and practices of nursing personnel regarding advance directives. International Journal of Palliative Nursing, 2007. 13(3): p. 109-115.

[52] Chan, C.W.H., et al., A systematic review of the effects of advance care planning facilitators training programs. BMC Health Services Research, 2019. 19(1): p. 362.

[53] Oczkowski, S.J., et al., Communication tools for end-of-life decision-making in ambulatory care settings: a systematic review and meta-analysis. PloS one, 2016. 11(4): p. e0150671. 
[54] L., R.B., et al., Initiating Advance Care Planning in Primary Care: A Model for Success. Journal of Palliative Medicine, 2019. 22(4): p. 427-431.

[55] Pettit, J.M., et al., Medical-Legal Partnerships to Enhance Residency Training in Advance Care Planning.

[56] Finn, L. and S. Malhotra, The Development of Pathways in Palliative Medicine: Definition, Models, Cost and Quality Impact. Healthcare (Basel), 2019. 7(1).

[57] Association, E.P. Care Pathways. Available from: e-p-a.org/care-pathways.

[58] Arnett, K., et al., Advance Care Planning: Understanding Clinical Routines and Experiences of Interprofessional Team Members in Diverse Health Care Settings. The American journal of hospice \& palliative care, 2017. 34(10): p. 946-953.

[59] Mortimer, L., et al., Centers for Medicare \& Medicaid Services' Oncology Care Model. Clinical Pharmacology \& Therapeutics, 2017. 101(5): p. 569-571.

[60] Bestvina, C.M. and B.N. Polite, Implementation of advance care planning in oncology: A review of the literature. Journal of oncology practice, 2017. 13(10): p. 657-662.

[61] Jimenez, G., et al., State of advance care planning research: A descriptive overview of systematic reviews. Palliative and Supportive Care, 2018. 17(2): p. 234-244.

Citation: Laura Finn et al. Advance Care Planning in Healthcare Systems: A Literature Review and Call to Action. ARC Journal of Nursing and Healthcare. 2019; 5(3):34-41. doi: dx.doi.org/10.20431/2455-4324.0503005.

Copyright: (C) 2019 Authors. This is an open-access article distributed under the terms of the Creative Commons Attribution License, which permits unrestricted use, distribution, and reproduction in any medium, provided the original author and source are credited. 